\title{
Excellence in cost-effective inpatient specialist palliative care in the NHS - a new model
}

\author{
Authors: Eleanor Grogan, ${ }^{A}$ Paul Paes ${ }^{B}$ and Tim Peel ${ }^{C}$
}

\begin{abstract}
There is little in the literature describing hospital specialist palliative care units (PCUs) within the NHS. This paper describes how specialist PCUs can be set up within and be entirely funded by the NHS, and outlines some of the challenges and successes of the units. Having PCUs within hospitals has offered patients increased choice over their place of care and death; perhaps not surprisingly leading to a reduced death rate in the acute hospital. However, since the opening of the PCUs there has also been an increased home death rate. The PCUs are well received by patients, families and other staff within the hospital. We believe they offer a model for excellence in cost-effective inpatient specialist palliative care within the NHS.
\end{abstract}

KEYWORDS: Specialist palliative care unit, cost effective, death rate

\section{Background}

Northumbria Healthcare NHS Foundation Trust is an innovative trust delivering hospital, community health and some adult social care services to a population of just over 500,000 people in North Tyneside and Northumberland. It covers one of the largest geographical areas of any trust in England. The hospital service is delivered through three general hospitals and seven community hospitals with a total of approximately 1,200 beds. A full range of community services are provided in both health settings and people's homes to both urban and rural populations.

Prior to 2009, specialist palliative care services for this population were patchy, characterised by a lack of service provision and investment. There was no inpatient palliative care provision, patients instead having to travel out of area to one of two hospices. This was reflected in a higher than average hospital death rate and lower than average hospice death rate. ${ }^{1}$

The death rates in the tables do not add up to all deaths within the Northumbria Healthcare catchment area as some patients

Authors: ${ }^{\text {A }}$ consultant and honorary clinical senior lecturer, Palliative Medicine, Wansbeck General Hospital, Ashington, UK; ${ }^{B}$ consultant and honorary clinical senior lecturer, Palliative Medicine North Tyneside General Hospital, North Shields, UK; ${ }^{C}$ consultant, Palliative Medicine, North Tyneside General Hospital, North Shields, UK die in other geographical areas. The trust embarked on a significant programme of investment and palliative care service development to develop a full, integrated service across all settings. With the background of high rates of hospital deaths and relatively low hospice use due to the distance from our local hospices, it was decided the first step was to develop NHS inpatient specialist palliative care facilities in the two main general hospitals of Northumbria Healthcare. These are North Tyneside General Hospital and Wansbeck General Hospital which is in Northumberland. There is little in the literature describing hospital specialist palliative care units (PCUs) within the NHS, although papers from the USA demonstrate that having dedicated PCUs can reduce healthcare costs. ${ }^{2,3}$ In the UK, there are a small number of these units, with a larger number of NHS hospices as part of hospital organisations.

\section{Development of the palliative care units}

Prior to 2009, epidemiological needs assessments had suggested an increase in palliative care bed provision was required. This was confirmed in 2009 by a series of point prevalence surveys, identifying the number of 'palliative patients' at any one time. This estimated a need for 15-20 beds in each of the main hospitals. There was no new money so the budgets for creating the PCUs came from closing acute medical beds and switching the resource to delivering specialist palliative care beds. Both PCUs are entirely NHS funded - charitable monies received are relatively modest and are used to purchase luxuries for patients, for example complementary therapies.

North Tyneside General Hospital Palliative Care Unit (NTPCU) opened in December 2009. The location chosen was within the hospital grounds, leasing the first-floor wing of a newly built nursing home. It has 19 single rooms with en-suite toilets and was modified to meet hospital ward specifications. The unit is staffed by trust employees, with catering provided by the nursing home.

Wansbeck General Hospital Palliative Care Unit (WPCU) opened in December 2012. Unlike its sister unit, WPCU is based in the main hospital, on the main corridor. It has twenty beds - two bays of six beds, one bay of three beds and five single rooms. All staffing is provided by trust employees, at usual trust ratios (rather than the higher level of many specialist PCUs or hospices) with non-medical staff paid under the NHS Agenda for Change agreement. As well as medical, nursing and allied health professional staff, both PCUs have regular support 
from chaplaincy and pharmacy, plus liaison psychiatry and psychology as requested. Both PCUs are funded as per an acute medical bed tariff.

\section{Activity}

There is a difference between the activity data of the units. NTPCU has less admissions overall, more direct community admissions (including from clinics) and a slightly longer length of stay. Data from 2014/2015 demonstrate a mean length of stay of 13 days at NTPCU and 12 days at WPCU. Most admissions to WPCU come from acute wards, particularly the medical admissions unit (probably because both are within the main hospital). The death rate is around $50 \%$ in both units, similar to other specialist PCUs.

We think that patients coded as 'non-palliative care patients' or 'boarders' may have been in error at NTPCU as no nonpalliative patients are admitted there. Two of the three patients coded as boarders died on the PCU, confirming this suspicion.

The number of 'boarders' at WPCU may have been higher than actual for different reasons - patients transferred from other wards are often initially coded as 'boarders' as ward clerks do not make the decision that a patient is palliative. Since this has been recognised, consultants are working with the ward clerks to improve coding. The figures for the number of patients admitted and died or discharged are not equal because there is always ongoing patient movement. Also, occasional patients are transferred to other wards.

\section{Death rates}

For the first time in our area, patients have the choice to be admitted to a specialist palliative care inpatient bed locally rather than face the choice of travelling, being in an acute bed locally or struggling at home. Place of death data have been transformed with a notable increase in death of usual place of residence. This is combined with an increase in deaths in palliative care settings and a reduction in acute hospital deaths, as shown in Table 1. ${ }^{1,4}$

\begin{tabular}{|c|c|c|c|}
\hline \multirow[t]{2}{*}{ Place of death } & $2008 / 9$ & $2008 / 9$ & $2013 / 14$ \\
\hline & $\begin{array}{l}\text { Northumbria } \\
\text { Healthcare NHS } \\
\text { Foundation } \\
\text { Trust, \% }\end{array}$ & $\begin{array}{l}\text { England } \\
\text { average, } \\
\%\end{array}$ & $\begin{array}{l}\text { Northumbria } \\
\text { Healthcare NHS } \\
\text { Foundation } \\
\text { Trust, \% }\end{array}$ \\
\hline $\begin{array}{l}\text { Usual place of } \\
\text { residence - North } \\
\text { Tyneside }\end{array}$ & 39.1 & 38.1 & 50.3 \\
\hline $\begin{array}{l}\text { Usual place } \\
\text { of residence - } \\
\text { Northumberland }\end{array}$ & 39.3 & & 44.8 \\
\hline Acute Hospital & 40.6 & 54.5 & 31.1 \\
\hline PCU & 0.0 & & 8.6 \\
\hline Hospice & 2.1 & 5.2 & NA \\
\hline
\end{tabular}

$\mathrm{NA}=$ not available; $\mathrm{PCU}=$ palliative care unit.

\section{Discussion}

\section{Contrast between palliative care unit and NHS hospice}

Although the PCUs and some NHS hospices are both in hospital settings, a deliberate decision was made to base the model on a hospital ward, rather than to seek extra funding through the NHS or fundraising to increase the staffing or create a purpose built facility. We believed that a high level of care could be delivered on that basis, allowing any extra money coming into the service to focus on community-based care. Many NHS hospices started off initially as hospital palliative care wards or units. Our intention is not to go down that route because we feel there is a danger that a high-profile hospice would divert money and focus towards inpatient care, which is contrary to our community-based model of care.

\section{Location}

The two contrasting locations on an acute site provide both advantages and disadvantages. WPCU is located on the main hospital corridor, is at the hub of the hospital and, as such, has a high profile. An on-site PCU allows patients to be easily transferred, including those with acute unstable conditions or from the intensive care unit. They can also be easily moved for investigations. On the other hand, there is less control over beds by PCU staff. The trust admissions policy means that if the hospital is under pressure for beds, WPCU becomes vulnerable to patients from other specialties being looked after on the PCU as a 'boarder'. This limits the ability to take direct admissions to the PCU. However, it keeps up the level of general medicine skills and means that medical staff from other specialities see the type of care and complexity of patients, helping to dispel the myth that patients are only admitted for end-of-life care.

In contrast, NTPCU is off the main hospital site, though only a five-minute walk. This has allowed the PCU to develop as a

\section{Table 2. Current PCU staffing levels.}

\begin{tabular}{|c|c|c|}
\hline Staff & NTPCU & WPCU \\
\hline Consultants & $\begin{array}{l}0.5 \text { WTE - } 2 \\
\text { individuals }\end{array}$ & 0.5 WTE -2 individuals \\
\hline $\begin{array}{l}\text { Training } \\
\text { doctors }\end{array}$ & $\begin{array}{l}\text { 2.4 WTE - } 1 \text { CMT and } \\
1.4 \text { GPST }\end{array}$ & $\begin{array}{l}2.4 \text { WTE - } 1 \mathrm{FT}, 1 \mathrm{CMT} \\
\text { and } 0.4 \mathrm{GPST}\end{array}$ \\
\hline Nursing staff & $\begin{array}{l}3 \text { early shift; } 2 \text { late } \\
\text { shift; } 2 \text { night shift }\end{array}$ & $\begin{array}{l}3 \text { early shift; } 2 \text { late } \\
\text { shift; } 2 \text { night shift }\end{array}$ \\
\hline $\begin{array}{l}\text { Health care } \\
\text { assistants }\end{array}$ & $\begin{array}{l}2 \text { early shift; } 2 \text { late } \\
\text { shift; } 2 \text { night shift }\end{array}$ & $\begin{array}{l}3 \text { early shift; } 2 \text { late } \\
\text { shift; } 2 \text { night shift }\end{array}$ \\
\hline Physiotherapy & 0.5 WTE & 0.5 WTE \\
\hline $\begin{array}{l}\text { Occupational } \\
\text { therapy }\end{array}$ & 0.5 WTE & 1 WTE \\
\hline Social work & 1 WTE & 0.5 WTE \\
\hline Domestic staff & $\begin{array}{l}1 / 2 \text { morning; } \\
1 \text { afternoon }\end{array}$ & $\begin{array}{l}1 \text { on each shift: } 7 a m- \\
1 p m ; 9 a m-3 p m ; 2 p m \\
-8 p m\end{array}$ \\
\hline
\end{tabular}


Table 3. PCU activity analysis 2014/15.

NTPCU WPCU

Palliative care patients

\begin{tabular}{lll} 
Direct admissions & 173 & 106 \\
Transfers to PCU & 246 & 386 \\
Total palliative PCU admissions & 419 & 492 \\
Discharges to usual place of residence & 181 & 184 \\
Discharge to other provider & 3 & 8 \\
Deaths & 203 & 253 \\
Total discharges and deaths & 387 & 445 \\
Bed days & 5,223 & 6,054 \\
Non-palliative care patients or 'boarders' & & \\
Patients, n & 4 & 184 \\
Bed days & 29 & 508 \\
\hline
\end{tabular}

NTPCU $=$ North Tyneside General Hospital Palliative Care Unit; $P C U=$ palliative care unit; WPCU = Wansbeck General Hospital.

more independent service with full control over beds and no 'boarders', allowing continuous direct admissions. However, some of the more acute or unstable patients spend longer on an acute ward than they would at WPCU. Most investigations require the patient to be taken in an ambulance to the main site because it is not physically connected. There are sometimes delays in other professionals visiting NTPCU if an opinion is requested. Because NTPCU was originally designed as a nursing home, patients benefit from single rooms with associated privacy and dignity. Conversely, there is no piped oxygen and both the lift and doorways were not designed for hospital beds which can inhibit patient transfers.

Both PCUs have a day room and kitchen facilities that can be accessed by patients and relatives.

\section{Staffing}

In both PCUs, the nursing staff were recruited to create a new team, with modern matrons to support the ward managers. Some had significant palliative care experience which was crucial in the early days. The skills of the team have grown over time as experience has been built up, and will continue to develop. Staffing levels could be higher, which may improve care, but the perceived quality of care being delivered (as evidenced by patient experience data - see later) makes it hard to argue that the PCUs need greater staffing than in other areas of the hospital. Staff satisfaction and sickness absence have been very good.

Junior doctors rotate every 4-6 months, which can influence the quality of care. There are posts for foundation 2 , core medical trainee and general practitioner vocational training scheme doctors, providing many junior doctors the opportunity to work in a specialist palliative care environment and develop skills to take with them in their career. These posts have become stepping stones on to higher specialist training. Largely this has worked well, but a permanent middle-grade doctor or nurse practitioner may help overcome the flux for other more permanent staff. Initially, physiotherapists and occupational therapists rotated into the PCUs which had the advantage of up-skilling the pool of hospital physiotherapists and occupational therapists, but was less stable for the PCUs. The PCUs are now moving towards having permanent physiotherapists and occupational therapists who can develop more specialised knowledge and skills. There are permanent social workers who are crucial to patient and family support, as well as discharge planning.

\section{Day-to-day working}

The PCUs both have their own morning multidisciplinary handover meeting but do not have a scheduled admissions meeting, preferring to accept referrals at any time of the day. Direct community admissions occur regularly during the working day but are more limited out of hours in that they require a doctor to be present on the ward to admit them. Patients can be transferred from within the hospital at any time, although this is usually done within the daytime or evening to avoid night-time disruption for patients. A single referral form is used for all palliative care services within Northumbria healthcare, including the PCUs. However, the PCUs are happy to accept referrals in any format - the ethos is to try and make the process as easy as possible for referrers, patients and their families.

\section{Out-of-hours medical cover}

Palliative medicine consultants provide a second tier of on-call cover and review patients on the PCUs seven days a week to assess complex, unstable patients or new admissions, as well as regular ward rounds. The first tier of on-call comes from the general medicine on-call team. This team covers a number of areas meaning there is not capacity for out-of-hours direct admissions; instead patients go via the medical assessment units for an initial assessment prior to transfer to the PCUs. At WPCU, out-of-hours cover can be present quickly if patients require rapid assessment. At NTPCU the off-site location makes this more challenging, because the on-call team have a distance

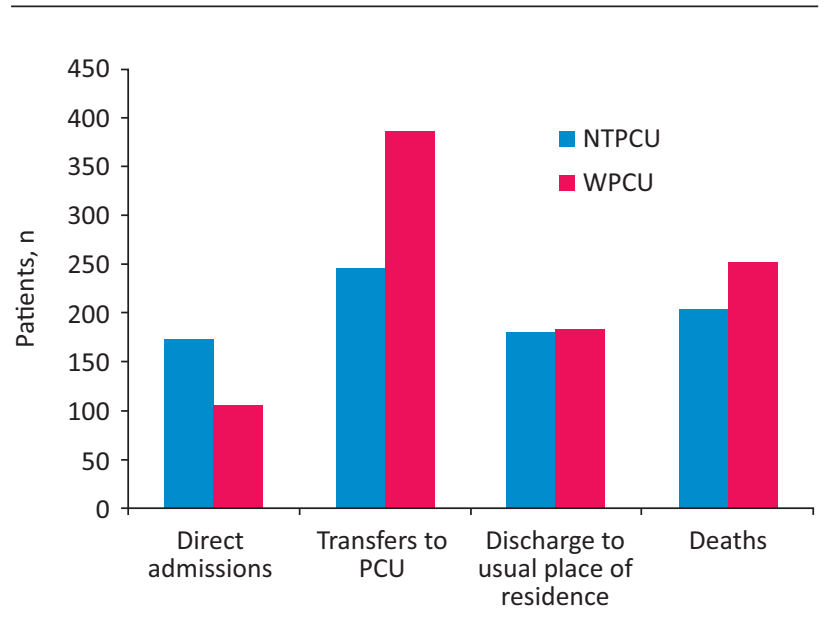

Fig 1. PCU activity analysis 2014/2015. NTPCU = North Tyneside General Hospital Palliative Care Unit; PCU = palliative care unit; WPCU = Wansbeck General Hospital. 
Fig 2. Place of death. NTPCU opened December 2009; WPCU (in Northumberland) opened December 2012. NTPCU = North Tyneside General Hospital Palliative Care Unit; $\mathrm{PCU}=$ palliative care unit; $\mathrm{WPCU}=$ Wansbeck General Hospital.

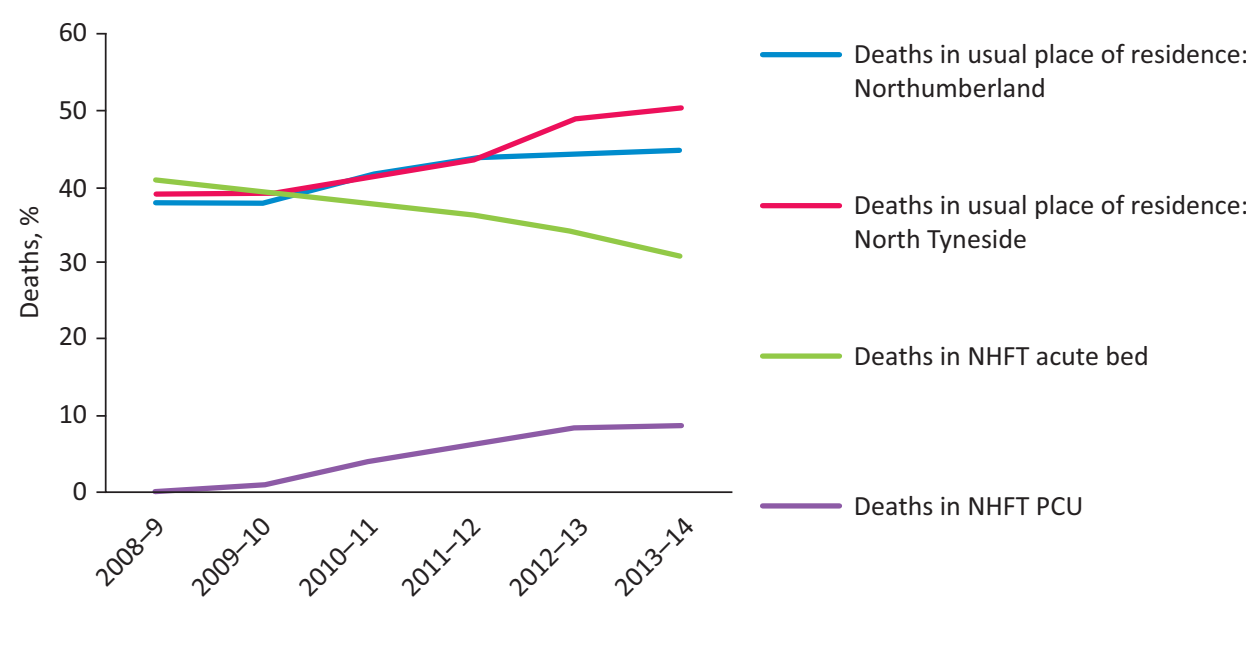

to come. These problems will be overcome by our plans to have on-call nurse practitioners which will enable direct admissions and more timely input.

\section{Patient monitoring and observations}

Both PCUs adhere to the quality assessments that apply to all wards within the trust, such as falls risk and pressure area assessments. One difference is the application of the NEWS scoring system. As part of individualised care plans, palliative medicine consultants decide whether, for a given patient, National Early Warning Score (NEWS) (in other words, regular observations of temperature, blood pressure, pulse, respiratory rate and oxygen saturations) should be measured, and if so how frequently. For many patients, a decision is made not to do this regularly as it would not alter their care. However, if the nursing staff notice an unexpected change they would perform a NEWS and report this to a doctor if required. By not performing regular observations on all patients, a large amount of nursing and healthcare assistant time is freed up.

\section{Treatment escalation}

As a part of an acute trust, and with out-of-hours junior medical cover coming from the general medical on-call team, it has been important to try and anticipate clinical problems that may arise and set clear expectations around escalations of care. A treatment escalation plan document (see supplemental material, S1) has been developed and is now being used throughout the trust to anticipate possible deteriorations in a patient's condition and set appropriate levels of care. These are done in conjunction with the patients, and often their family, usually on admission to the PCU (or increasingly to the medical admissions unit) or as their condition evolves.

\section{Visiting policy}

Both PCUs have open visiting hours, unlike most other wards in the trust. This initially caused some apprehension among staff but it has been well received. Doctors can see patients with their families, so ward rounds can include both the patient and those important to them, if they so wish. There is always the time for private discussions if the patient prefers. Nurses and healthcare assistants plan their work around visitors and there are spaces on both PCUs for families to sit with refreshments while patients are attended to.

\section{Feedback and profile}

The trust performs monthly patient experience feedback by assessing user satisfaction in many of its wards. Patients and/ or carers complete a questionnaire with a clerical officer, independent of the ward, and score various parameters of their care. The PCUs are consistently the highest scoring in the trust and the first to score 10/10 in all domains measured.

In 2102 the PCUs participated in the Association for Palliative Medicine pilot audit of bereaved carers' satisfaction with the care received, using the FAMCARE 2 tool. ${ }^{4}$ It was repeated the following year, demonstrating improved scores, particularly in issues of communication with the family.

In the 2014 trust annual awards, the PCUs won the quality award. Mortality audits are carried out throughout the trust and highlight the quality of end-of-life care being delivered on the PCUs. This high profile has led to a greater priority for palliative and end-of-life care issues. When the PCUs were developed, there was a concern that the role and quantity of work of the hospital palliative care teams would reduce. In fact, the increased profile has led to a greater number of referrals and workload, including more complex shared care patients.

\section{Palliative care units as preferred place of care}

Patients now know about the PCUs, and many are choosing it as their place of care and death. This now means that capacity is sometimes unable to meet demand, resulting in a waiting list which we try hard to avoid. Sometimes patients' expectations are not aligned with healthcare professionals in terms of whether they are stable enough to be discharged, highlighting the tension between preferred place of care and what is realistic, the commissioning drive for greater community care and the challenges for community teams to look after complex patients at home. 


\section{Summary}

Dedicated NHS specialist PCUs within a hospital funded by the NHS at usual ward levels:

$>$ have led to an increase in home death rate

$>$ have led to a reduction of deaths in the acute hospital setting

$>$ have led to an increase in death rate in a specialist palliative care setting

$>$ can be delivered at a significantly lower cost than setting up a more conventional hospice in a hospital or community setting, and with lower staffing levels than many such units

$>$ have led to better integration of community, hospital and PCU teams, facilitating ease of admissions and discharges home for patients

> allow patients to be admitted directly to the palliative care setting, rather than via the acute route with an associated cost reduction and improvement in patient experience

$>$ are well received by patients, relatives, staff within and without the palliative care setting

$>$ have raised the profile of palliative care within the trust.

The work done within Northumbria Healthcare NHS

Foundation Trust over the last few years demonstrates that it is possible to achieve excellence and cost-effective inpatient specialist palliative care within the NHS. This has been done with a contrasting model of care to a more conventional hospice inpatient service, and may be of particular interest to those areas where the cost of inpatient hospice care is prohibitive or a more integrated model is desired.

\section{References}

1 Network National End-of-Life Care Intelligence. Local Authority Profiles 2012. London: PHE, 2012. Available online at www.endoflifecare-intelligence.org.uk/end_of_life_care_profiles/la_2012_pdfs [Accessed 18 December 2015].

2 Smith TJ, Coyne P, Cassel B et al. A high-volume specialist palliative care unit and team may reduce in-hospital end-of-life care costs. J Palliat Med 2003;6:699-705.

3 Elsayem A, Swint K, Fisch MJ et al. Palliative care inpatient service in a comprehensive cancer center: clinical and financial outcomes. J Clin Oncol 2004;22:2008-14.

4 Network National End-of-Life Care Intelligence. Place of death. London: PHE, 2013. Available online at www.endoflifecare-intelligence. org.uk/data_sources/place_of_death [Accessed 18 December 2015].

5 Beaumont C, Nekolaichuk C. FAMCARE and FAMCARE-2 guidelines for use. Edmonton, AB: Palliative and End-of-Life Care Institute, 2012. Available online at http://palliative.org/NewPC/ pdfs/tools/FAMCARE\%20Guidelines_v2\%200_12Dec2012\%20(2). pdf [Accessed 11 November 2015].

Address for correspondence: Dr E Grogan, Palliative Medicine, Wansbeck General Hospital, Woodhorn Lane, Ashington NE63 9J], UK.

Email: eleanor.grogan@nhct.nhs.uk 